\title{
Left Atrial Dyssynchrony in Patients With Nonobstructive Hypertrophic Cardiomyopathy Evaluated by Myocardial Strain Imaging
}

\author{
Yasunori Kawanishi, MD*, Takahide Ito, MD*, Rie Futai, MD*, Akio limori, MD*, \\ Akira Ukimura, MD*, Koichi Sohmiya, MD*, Fumio Terasaki, MD*, \\ Michihiro Suwa, MD** and Yasushi Kitaura, MD* \\ *Third Department of Internal Medicine, Osaka Medical College, Takatsuki, Japan \\ **Department of Cardiovascular Medicine, Hokusetsu General Hospital, Takatsuki, Japan
}

\begin{abstract}
Background. The present study was conducted to examine left atrial (LA) dyssynchrony, determined by echocardiographic myocardial strain imaging, in patients with hypertrophic cardiomyopathy (HCM).

Methods. We studied 26 patients with HCM (20 men and 6 women, age, $66 \pm 7$ years) and 25 normal subjects (controls). Tissue Doppler-based strain images were recorded in the apical 2-, 4-chamber, and long-axis views. The LA strain curve was obtained with the region of interest placed at the 5 LA segments, namely, the atrial septum, lateral wall, inferior wall, anterior wall, and posterior wall. We measured standard deviation (SD) of the time-periods between aortic valve opening and maximal strain (reservoir dyssynchrony index) and SD of the time-periods between mitral valve opening and minimal strain (emptying dyssynchrony index).

Results. Compared with controls, patients with HCM had greater indexes of reservoir $(40 \pm 23 \mathrm{~ms} v \mathrm{vs} .23 \pm 8 \mathrm{~ms}$, $\mathrm{p}=0.001)$ and emptying ( $37 \pm 17 \mathrm{~ms}$ vs. $28 \pm 11 \mathrm{~ms}, \mathrm{p}=0.02)$ dyssynchrony. Reservoir dyssynchrony (index $>39 \mathrm{~ms}$ ) was detectable in 11 patients, and emptying dyssynchrony (index $>50 \mathrm{~ms}$ ) was detectable in 5 patients. Patients with reservoir dyssynchrony had a greater LV mass index than those without $\left(182 \pm 45 \mathrm{~g} / \mathrm{m}^{2} \mathrm{vs} .142 \pm 32 \mathrm{~g} / \mathrm{m}^{2}, \mathrm{p}=0.01\right)$, whereas patients with emptying dyssynchrony had greater LA minimal volume $\left(39 \pm 12 \mathrm{ml} / \mathrm{m}^{2} \mathrm{vs} .26 \pm 10 \mathrm{ml} / \mathrm{m}^{2}\right.$ $\mathrm{p}=0.02)$ and lower LA emptying fraction than those without $(27 \pm 3 \%$ vs. $39 \pm 11 \%, p=0.02)$.

Conclusions. LA dyssynchrony can be present throughout the cardiac cycle in patients with HCM. LA dyssynchrony is more common in the reservoir phase and may be attributed to significant myocardial involvement.
\end{abstract}

(J Echocardiogr 2008; 6: 46-53)

Key words: hypertrophic cardiomyopathy, atrial function, dyssynchrony, strain imaging

\section{Introduction}

Increased chamber stiffness resulting from left ventricular (LV) hypertrophy can be a systolic and diastolic burden on the left atrial (LA) chamber, whereas LA function is of clinical importance for maintaining

Received April 2, 2008; revision received May 14, 2008; accepted June 5, 2008

Address for correspondence: Yasunori Kawanishi, MD

Third Department of Internal Medicine, Osaka Medical College,

2-7, Daigaku-cho, Takatsuki, 569-8686, Japan.

Telephone: +81-72-683-1221 (ext. 2353)

Fax: +81-72-683-6597

E-mail: in3087@poh.osaka-med.ac.jp

(C) 2008 Japanese Society of Echocardiography adequate LV filling and forward output. Hypertrophic cardiomyopathy (HCM) has an extremely hypertrophied LV and is commonly associated with LA enlargement and dysfunction [1,2]. Numerous echocardiographic studies have investigated LA function in patients with a variety of heart diseases [1-8]. In patients with $\mathrm{HCM}$, it is generally accepted that increased LA volume is associated with a higher incidence of LV diastolic dysfunction and cardiac events and more severe LV hypertrophy [2,4]. A lack of organized LA relaxation and contraction in these patients may lead to hemodynamic derangement [2]. Tissue Doppler imaging is the most commonly used technique for defining LV systolic dyssynchrony [9]. Previous studies of tissue Doppler imaging demon- 
strated that the degree of LV dyssynchrony was pronounced proportionally with the severity of the myocardial disease process [9-11]. Given the common finding of LA enlargement, we currently hypothesized that HCM patients might also have LA dyssynchrony. Myocardial strain imaging provides indexes of myocardial deformation in response to an applied force. This technique is theoretically independent of cardiac translational motion and tethering, allowing differentiation between active and passive myocardial motion [12,13]. It is also able to assess the timing of systolic and diastolic events of the cardiac chamber $[14,15]$. The purpose of the present study was to examine LA systolic (emptying) and diastolic (reservoir) dyssynchrony using myocardial strain imaging in patients with $\mathrm{HCM}$ and to determine its incidence and possible relationship with other echocardiographic parameters.

\section{Methods}

\section{Study population}

We studied 26 patients with HCM (20 men and 6 women, age, $66 \pm 7$ years) and 25 normal healthy individuals (10 men and 15 women, age, $63 \pm 11$ years). All patients were clinically stable and examined at the visit to the outpatient department. The diagnosis of HCM was based on the presence of a hypertrophied, nondilated LV chamber with a ratio of the ventricular septum to posterior wall thickness of at least 1.3. Patients who had a chamber obstruction, those with chronic atrial fibrillation, and those who had poor image quality were excluded. Twelve patients had been undergoing therapy for concomitant hypertension, and their blood pressure was well controlled; 8 were being treated with calcium-channel antagonists; 6 with
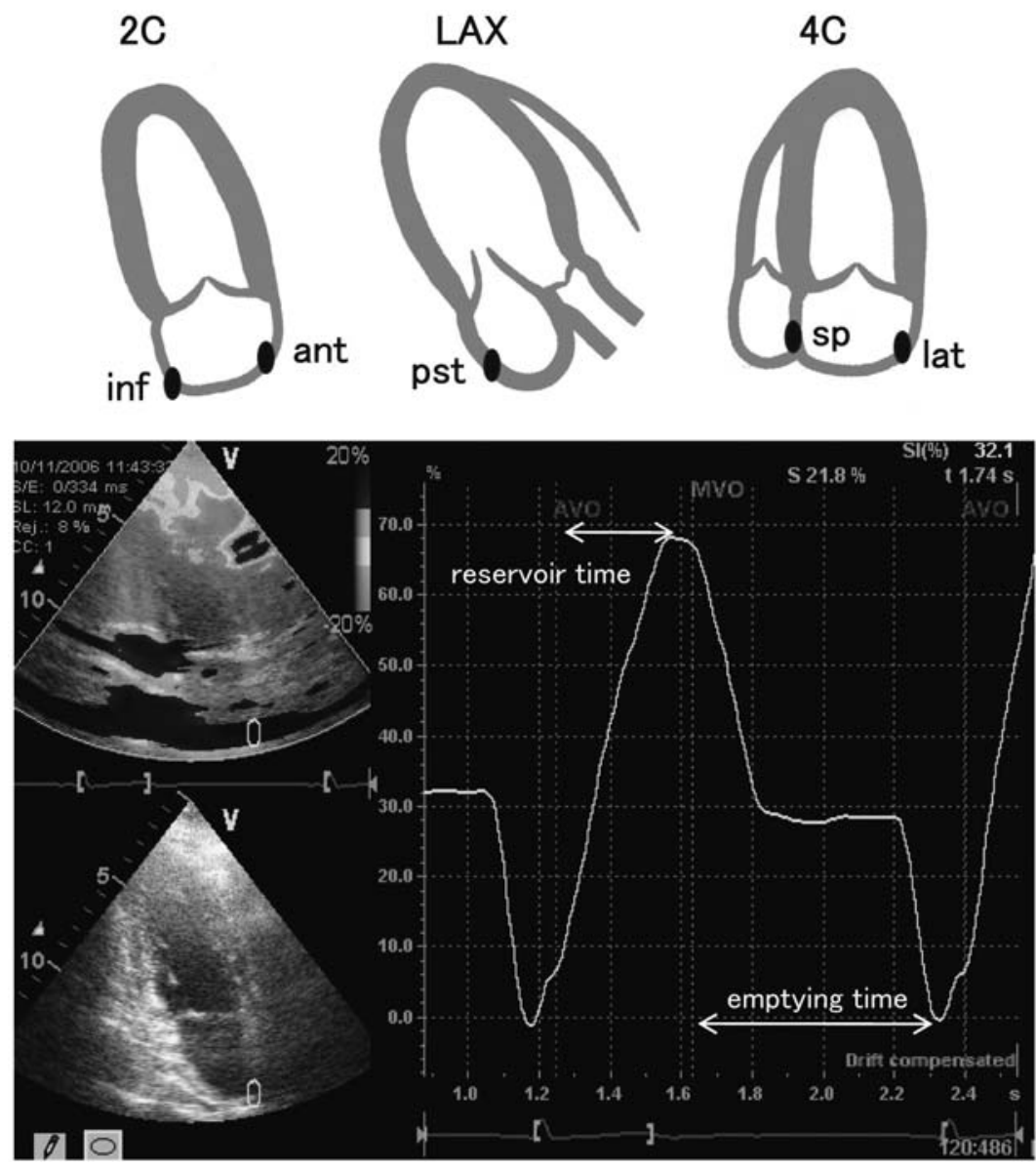

Fig. 1. Top, schema of apical 2-, 4- chamber and long-axis views, depicting left atrial myocardial segments to be analyzed; Bottom, a representative myocardial velocity curve arising from the anterior wall. AVC, timing of aortic valve closure; ant, anterior wall; inf, inferior wall; lat, lateral wall; MVO, timing of mitral valve opening; pst, posterior wall; sp, septum. 
angiotensin converting enzyme inhibitors; and 5 with angiotensin II receptor blockers. Normal subjects served as controls; they had no history of cardiovascular disease and showed normal findings on routine echocardiographic examination. Written informed consent was obtained from all subjects.

\section{Conventional echocardiography}

Standard echocardiography was performed using a commercially available equipment (Vivid 7 [GEVingmed Ultrasound, Horten, Norway]) with an M4s transducer. LA diameter was measured at early diastole along the parasternal long-axis view with 2dimensinonal guided M-mode tracing. LV systolic and diastolic dimensions and wall thickness were also calculated. LV ejection fraction was determined by the modified Simpson's method. LV mass was calculated as proposed by Devereux and Reichek [16] and indexed for the body surface area. Parameters of LV diastolic function were assessed by the pulsed Doppler method. These included LV inflow velocities of peak early (E) and atrial filling (A) waves, their ratio (E/A), and the $\mathrm{E}$ wave-deceleration time. Mitral regurgitation was semi-quantitatively graded using the color-Doppler method. Using tissue Doppler imaging, peak wall motion velocities of systolic (Sa), early diastolic (Ea), and atrial contraction $(\mathrm{Aa})$ waves were obtained with the sample volume placed at the septal corner of the mitral annulus. The E/Ea was calculated as a surrogate for LV filling pressure [17].

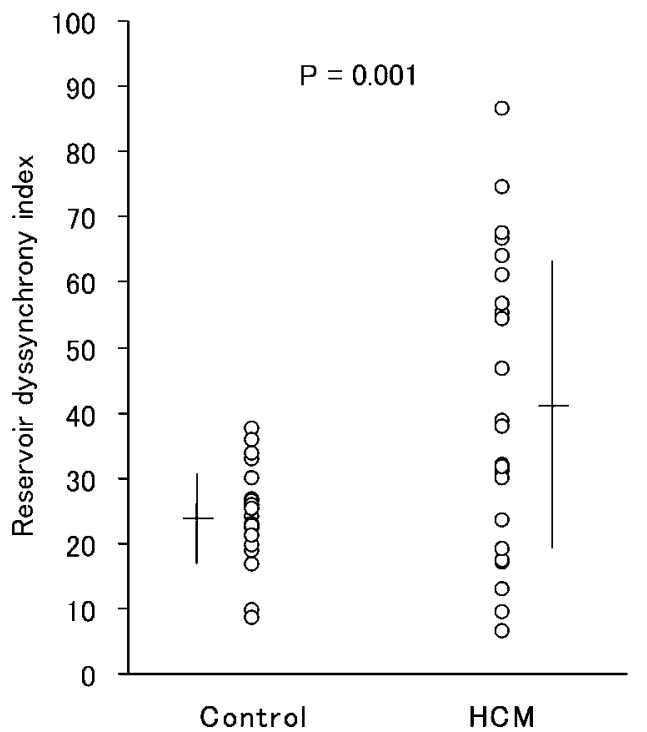

\section{Acquisition of myocardial strain data}

Tissue Doppler images, or color Doppler myocardial images, were recorded from the apical 2-, 4-chamber, and long-axis views such that the entire LA cavity was visible on the screens by adjusting the depth control. Gain settings, filters, and pulse repetition frequency were adjusted to optimize color saturation, and the sector size and depth were optimized for the highest possible frame rate ( $>140$ frames/s). At least three consecutive beats were digitally stored for post-processing. Myocardial strain data were processed from tissue velocity imaging data and analyzed on the software program provided with the same echocardiographic machine. With a region of interest of $8 \times 4 \mathrm{~mm}$ and strain length of $8 \mathrm{~mm}$, LA longitudinal strain curves were constructed offline. The segments analyzed were the atrial septum and LA lateral wall (from the apical 4chamber view), LA inferior and anterior walls (from the apical 2-chamber view), and the LA posterior wall (from the long-axis view) (Figure 1).

\section{Analysis of LA function and dyssynchrony}

Maximal and minimal LA volumes at the timing of early diastole (mitral valve opening) and end-diastole (mitral valve closure), respectively, were calculated from apical 2- and 4-chamber zoomed views using the biplane disk method [4]. These measurements were indexed for the body surface area. To characterize cardiac-cycle phases of LA cavity size and function, the total LA emptying volume and fraction were measured

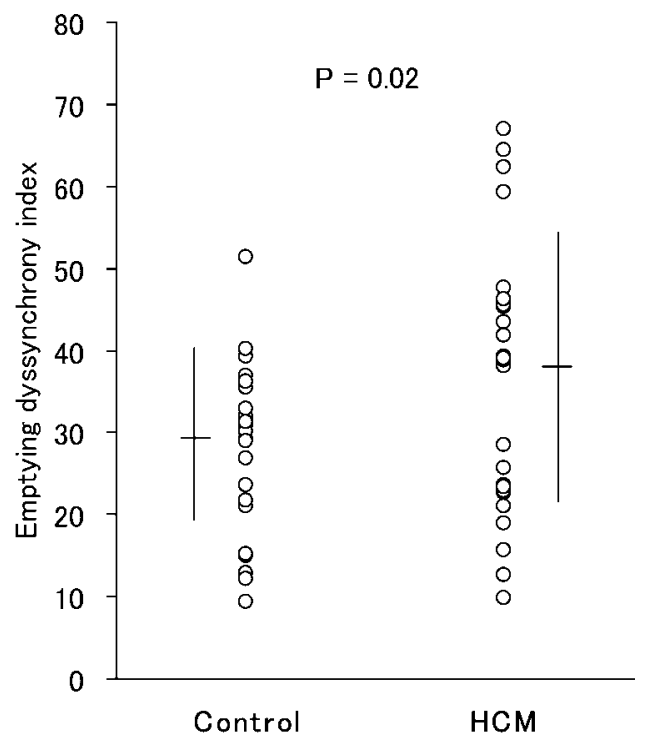

Fig. 2. Comparisons of the left atrial reservoir dyssynchrony index (left) and emptying dyssynchrony index (right) between the patients with hypertrophic cardiomyopathy (HCM) and normal subjects (controls). 
using the difference between maximal and minimal LA volumes. From the LA strain curves for each segment, we measured as follows: standard deviation (SD) of the time-periods between the timing of aortic valve opening and maximal strain (reservoir dyssynchrony index); and SD of the time-periods between the timing of mitral valve opening and minimal strain (emptying dyssynchrony index) (Figure 1). The timings of aortic and mitral opening were determined by incorporating pulsed Doppler recordings of LV outflow and inflow, respectively.

\section{Observer variability}

Reproducibility for the measurements of the timedomain parameters was assessed in 10 randomly selected subjects. Interobserver variabilities of the time-period to maximal strain and the time period to minimal strain were $11 \pm 11 \mathrm{~ms}$ (correlation coefficient $[\mathrm{r}]=0.97)$ and $17 \pm 15 \mathrm{~ms}(\mathrm{r}=0.96)$, respectively; likewise, intraobserver variabilities were $14 \pm 10 \mathrm{~ms}$ $(\mathrm{r}=0.96)$ and $17 \pm 14 \mathrm{~ms}(\mathrm{r}=0.95)$, respectively.

\section{Statistical analysis}

All data are expressed as the mean \pm SD. Differences in patient characteristics between the groups were assessed using the unpaired t test or Welch's test (does not assume equal variances) for continuous variables; for categorical variables the chi-square test or Fisher's exact test was used. A p value $<0.05$ was considered significant.

\section{Results}

\section{Conventional echocardiographic parameters}

M-mode and Doppler echocardiographic parameters in patients with HCM and control subjects are summarized in Table 1. The patient group had more female subjects although their age was similar. Heart rate was comparable during the echocardiographic examination. According to disease characteristics, patients with HCM had thicker LV walls for both the ventricular septum and posterior wall. LV systolic and diastolic dimensions and LV ejection fraction were comparable.

Table 1. Conventional echocardiographic parameters in the study subjects

\begin{tabular}{lccc}
\hline & $\begin{array}{c}\mathrm{HCM} \\
(\mathrm{n}=26)\end{array}$ & $\begin{array}{c}\text { Control } \\
(\mathrm{n}=25)\end{array}$ & $\mathrm{p}$ Value \\
\hline Age (years) & $66 \pm 7$ & $63 \pm 11$ & $\mathrm{NS}$ \\
Female (n) & 6 & 15 & $\mathrm{NS}$ \\
Body surface area (m $\left.{ }^{2}\right)$ & $1.6 \pm 0.1$ & $1.6 \pm 0.1$ & $\mathrm{NS}$ \\
Heart rate (beats/min) & $64 \pm 12$ & $67 \pm 14$ & $\mathrm{NS}$ \\
LA diameter (mm) & $43 \pm 7$ & $34 \pm 5$ & $<0.001$ \\
Thickness of the septum (mm) & $16 \pm 3$ & $8 \pm 1$ & $<0.001$ \\
Thickness of the posterior wall (mm) & $10 \pm 2$ & $9 \pm 1$ & $<0.001$ \\
LV end-diastolic dimension (mm) & $49 \pm 5$ & $47 \pm 4$ & $\mathrm{NS}$ \\
LV end-systolic dimension (mm) & $31 \pm 5$ & $30 \pm 4$ & $\mathrm{NS}$ \\
LV fractional shortening (\%) & $38 \pm 8$ & $35 \pm 6$ & $\mathrm{NS}$ \\
LV ejection fraction (\%) & $67 \pm 10$ & $64 \pm 8$ & $\mathrm{NS}$ \\
LV mass index (g/m $\left.{ }^{2}\right)$ & $159 \pm 42$ & $84 \pm 19$ & 0.001 \\
E (cm/s) & $68 \pm 16$ & $67 \pm 16$ & $\mathrm{NS}$ \\
A (cm/s) & $69 \pm 23$ & $75 \pm 15$ & $\mathrm{NS}$ \\
E/A & $1.1 \pm 0.7$ & $0.9 \pm 0.3$ & $\mathrm{NS}$ \\
Deceleration time (ms) & $200 \pm 70$ & $209 \pm 29$ & $\mathrm{NS}$ \\
Sa (cm/s) & $9.1 \pm 1.3$ & $6.8 \pm 2.0$ & $<0.001$ \\
Ea (cm/s) & $5.3 \pm 1.0$ & $8.5 \pm 2.3$ & $<0.001$ \\
Aa (cm/s) & $7.6 \pm 2.1$ & $10.3 \pm 1.7$ & $<0.001$ \\
E/Ea & $13.5 \pm 5.0$ & $8.3 \pm 2.3$ & $<0.001$ \\
\hline
\end{tabular}

Values are expressed as the mean \pm SD.

HCM, hypertrophic cardiomyopathy; LA, left atrial; LV, left ventricular; E, peak velocity of the early filling wave; A peak velocity of the atrial filling wave; Sa, peak velocity of the systolic wave at the mitral annulus; Ea, peak velocity of the early diastolic wave at the mitral annulus; Aa, peak velocity of the atrial contraction wave at the mitral annulus; NS, not significant 
Table 2. Parameters related to LA function

\begin{tabular}{lccc}
\hline & $\begin{array}{c}\text { HCM } \\
(\mathrm{n}=26)\end{array}$ & $\begin{array}{c}\text { Control } \\
(\mathrm{n}=25)\end{array}$ & p Value \\
\hline Maximal LA volume index $\left(\mathrm{ml} / \mathrm{m}^{2}\right)$ & $44.7 \pm 14.6$ & $23.2 \pm 6.9$ & $<0.001$ \\
Minimal LA volume index $\left(\mathrm{ml} / \mathrm{m}^{2}\right)$ & $28.6 \pm 11.7$ & $11.0 \pm 4.5$ & $<0.001$ \\
LA emptying volume index $\left(\mathrm{ml} / \mathrm{m}^{2}\right)$ & $16.1 \pm 6.6$ & $12.2 \pm 3.6$ & 0.01 \\
LA emptying fraction (\%) & $37 \pm 11$ & $54 \pm 9$ & $<0.001$ \\
\hline
\end{tabular}

Values are expressed as the mean \pm SD.

Abbreviations are the same as in Table 1.

Table 3. Parameters related to LA dyssynchrony

\begin{tabular}{|c|c|c|c|}
\hline & $\begin{array}{l}\text { HCM } \\
(n=26)\end{array}$ & $\begin{array}{l}\text { Control } \\
(\mathrm{n}=25)\end{array}$ & $\mathrm{p}$ Value \\
\hline \multicolumn{4}{|c|}{ Time to maximal strain in LA segments (reservoir phase) } \\
\hline Atrial septum (ms) & $325 \pm 52$ & $342 \pm 39$ & NS \\
\hline Lateral wall (ms) & $329 \pm 55$ & $339 \pm 43$ & NS \\
\hline Anterior wall (ms) & $347 \pm 55$ & $347 \pm 41$ & NS \\
\hline Inferior wall (ms) & $337 \pm 65$ & $322 \pm 51$ & NS \\
\hline Posterior wall (ms) & $332 \pm 57$ & $331 \pm 43$ & NS \\
\hline Reservoir dyssynchrony index & $40 \pm 23$ & $23 \pm 8$ & 0.001 \\
\hline \multicolumn{4}{|c|}{ Time to minimal strain in LA segments (empty phase) } \\
\hline Atrial septum (ms) & $497 \pm 131$ & $495 \pm 124$ & NS \\
\hline Lateral wall (ms) & $504 \pm 132$ & $494 \pm 124$ & NS \\
\hline Anterior wall (ms) & $506 \pm 152$ & $495 \pm 121$ & NS \\
\hline Inferior wall (ms) & $506 \pm 142$ & $496 \pm 127$ & NS \\
\hline Posterior wall (ms) & $525 \pm 135$ & $507 \pm 134$ & NS \\
\hline Emptying dyssynchrony index & $37 \pm 17$ & $28 \pm 11$ & 0.02 \\
\hline
\end{tabular}

Values are expressed as the mean \pm SD.

Abbreviations are the same as in Table 1.

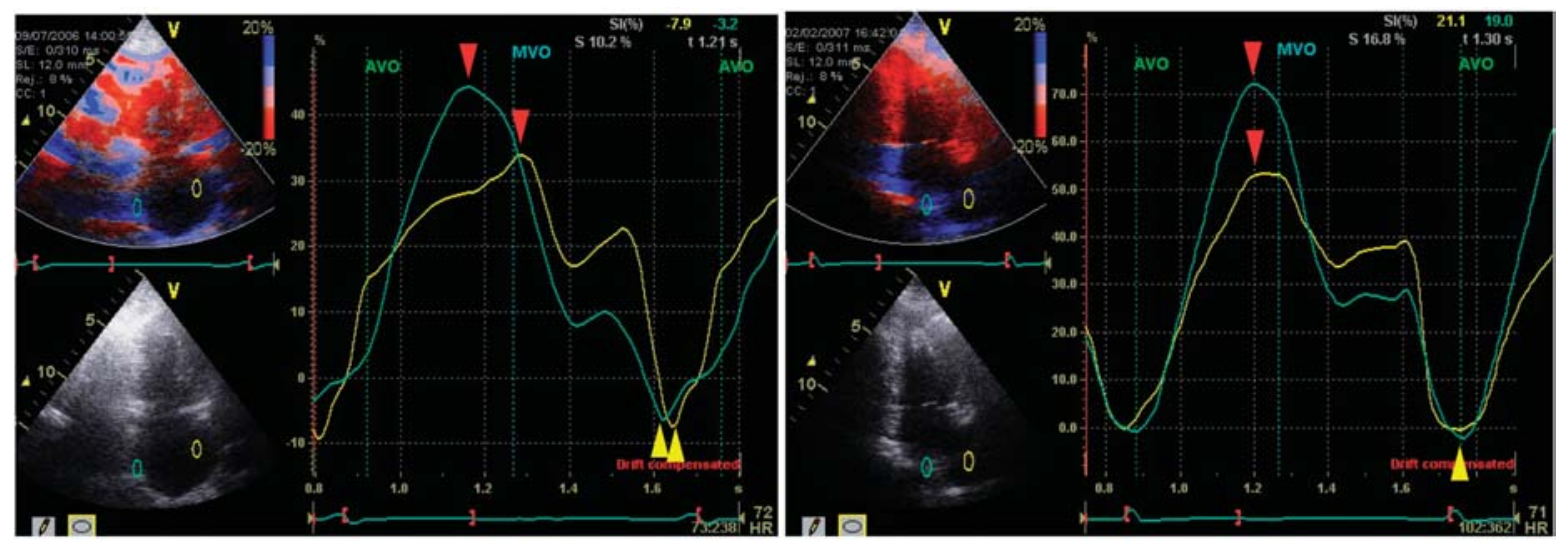

Fig. 3. Representative strain analyses of myocardial strain imaging for the anterior (yellow) and posterior (blue) left atrial (LA) segments from a patient with hypertrophic cardiomyopathy (HCM) (left) and a normal subject (right). Red arrowheads indicate the point of maximal LA strain and yellow the point of minimal LA strain. Abbreviations are as in Figure 1. 
Doppler parameters of LV inflow showed no significant differences, although patients with HCM showed significant decreases in $\mathrm{Ea}$ and $\mathrm{Aa}$, with a resultant increase in E/Ea. None of the patients had significant mitral regurgitation on color flow mapping.

\section{LA size and function}

The measurements of LA size and volume and its functional parameters are shown in Table 2 . Compared with controls, patients with HCM had greater LA diameter and maximal/minimal LA volumes. These findings were associated with significant larger LA emptying volume and less emptying fraction.

\section{LA dyssynchrony}

There were no significant differences between the two groups in either the time to maximal strain or the time to minimal strain for each segment (Table 3); however, the variation of the mean values was relatively larger in the HCM group, resulting in significant increases in both reservoir and emptying dyssynchrony indexes. Since $+2 \mathrm{SD}$ of the mean reservoir and emptying dyssynchrony index in controls was $39 \mathrm{~ms}$ and $50 \mathrm{~ms}$, respectively, LA reservoir dyssynchrony was defined as the index $>39$ ms and emptying dyssynchrony as the index $>50 \mathrm{~ms}$. Reservoir dyssynchrony was detectable in 11 patients (42\%) and emptying dyssynchrony in 5 patients (19\%). Patients with reservoir dyssynchrony were associated with greater ventricular septum thickness $(17 \pm 3 \mathrm{~mm}$ vs. $14 \pm 3 \mathrm{~mm}, \mathrm{p}=0.02)$ and $\mathrm{LV}$ mass index $\left(182 \pm 45 \mathrm{~g} / \mathrm{m}^{2}\right.$ vs. $142 \pm 32 \mathrm{~g} / \mathrm{m}^{2}$, $\mathrm{p}=0.01$ ) than those without. Patients with emptying dyssynchrony, on the other hand, showed greater LA minimal volume $\left(39 \pm 12 \mathrm{ml} / \mathrm{m}^{2}\right.$ vs. $26 \pm 10 \mathrm{ml} / \mathrm{m}^{2}$ $\mathrm{p}=0.02)$ and lower LA emptying fraction $(27 \pm 3 \%$ vs. $39 \pm 11 \%, p=0.02)$ than those without. No significant differences were found with regard to the parameters of LV systolic and diastolic function, including $\mathrm{E} / \mathrm{Ea}$, between patients with and without either type of LA dyssynchrony. Representative strain analyses are shown in Figure 3.

\section{Discussion}

\section{Main findings}

The results of this study demonstrated that compared with normal subjects, HCM patients had heterogeneous LA contraction and relaxation, as evidenced by increased indexes of LA dyssynchrony. Among patients with $\mathrm{HCM}, 42 \%$ were shown to have LA dyssynchrony in ventricular systole (reservoir dyssynchrony); that in ventricular diastole (emptying dyssyn- chrony) was less common (19\%). Such increases in the LA dyssynchrony indexes were associated with more severe LV hypertrophy, greater LA volume, as well as lower LA emptying fraction. To the best of our knowledge, this is the first study to examine LA dyssynchrony in heart disease patients based on timedomain analysis.

\section{Echocardiographic assessment of LA function in HCM}

Patients with HCM are known to have LA enlargement and dysfunction resulting from extreme LV hypertrophy [1,2]. Conventionally, LA size is measured by the modified Simpson's method [18]. The maximal LA volume in HCM patients was reported to be $50.1 \pm 15.9 \mathrm{ml} / \mathrm{m}^{2}$ compared with $30.1 \pm 6.8 \mathrm{ml} / \mathrm{m}^{2}$ in normal subjects [18]. These measurements were somewhat large compared with ours, although this discrepancy may be attributed to the differences in patient characteristics, including racial background. A previous study using the 2-dimensional method found that HCM patients had impaired LA systolic function, with a significant decrease in the LA emptying fraction [3]. Similar results were observed in another study that used real-time 3-dimensional echocardiography [4]. The authors found that, compared with normal subjects, HCM patients had increased LA volume, decreased LA active emptying fraction, and decreased maximal slope of the time-volume curve during early diastole, suggesting that increased LV stiffness might contribute to the impairment of LA function [4]. In a study of 2-dimensional speckle tracking, moreover, the LA radial strain was shown to be reduced in patients with idiopathic compared with ischemic dilated cardiomyopathy [5]. Since there was a similar degree of the parameters of loading conditions such as mitral flow velocity profiles, it was suggested that LA involvement in the myocardial process might contribute to LA myocardial dysfunction in idiopathic cardiomyopathy patients [5]. Only one study investigated regional LA function with strain or strain rate imaging in patients with HCM [6]. That study observed that in young patients, the longitudinal strain rate was reduced throughout the cardiac cycle at any levels of the LA wall compared with normal subjects and suggested that these changes of regional LA function might result from prolongation of LV relaxation [6]. Taken together, impairment of LV diastolic function, a hallmark of HCM, appears to play an important role in the development of LA enlargement and dysfunction. 


\section{Present observations}

In contrast to previous studies that examined LA myocardial strain, where strain measurements were carried out exclusively based on strain magnitude [68], the present study employed time-domain analysis. As it had been shown in previous studies [3,4], the present study found that, compared with controls, patients with HCM showed increased LA volumes and decreased LA systolic function (emptying fraction). As with LA dyssynchrony, it was currently observed that the derived indexes were increased throughout the cardiac cycle in the patients. In particular, LA dyssynchrony in ventricular systole was more common than that in diastole. The precise mechanism by which LA dyssynchrony develops is unclear. Previous investigations on LV dyssynchrony found that the incidence of systolic and diastolic LV dyssynchrony was lower in patients with preserved LV ejection fraction than in those with reduced LV ejection fraction $[9,19,20]$. Moreover, LV dyssynchrony following acute myocardial infarction was reported to predict more severe LV remodeling [21]. A similar relationship of the chamber size and function might be applied for the development of LA dyssynchrony. In the present study, patients with reservoir dyssynchrony had a greater LV mass index and wall thickness, whereas patients with emptying dyssynchrony had greater LA minimal volume and emptying fraction. Since the heart rate, mitral flow, and annular velocity profiles were similar between the subgroups of patients, it is reasonable to assume that LA in relation to LV myocardial involvement may contribute to the development of LA dyssynchrony. This hypothesis is further supported by previous studies demonstrating a positive correlation between LA size and LV wall thickness [3,4,22] and a higher degree of cell damage and fibrotic change in the thicker ventricular wall [23]. Moreover, it was recently reported in a study of 2-dimensional speckle tracking that patients with $\mathrm{HCM}$ had more severe LV dyssynchrony than those with hypertensive heart disease [24]. Since the ventricular systolic phase coincides with LA relaxation, there may be transmission of the LV myocardial contraction pattern to the thinwalled LA, with a resultant increase in the degree of reservoir dyssynchrony. In this study the incidence of reservoir dyssynchrony was more common than that of emptying dyssynchrony. This finding might be related to the fact that emptying dyssynchrony lies in an active process whereas reservoir dyssynchrony in the passive process, with the latter possibly being more susceptible to changes in the myocardial process.

\section{Limitations}

The present study has several limitations. We did not have information on hemodynamic data, which could have substantiated any contribution of LA pressure on LA functional parameters. The E/Ea, a represent noninvasive measure of LV filling pressure [11], however, did not differ between patients with and without LA dyssynchrony. In addition, assessment of pulmonary venous flow profiles could have complemented the interpretation of our data $[25,26]$, especially relationship between the systolic component and reservoir dyssynchrony. Some of the patients had hypertension, and thus possible influence of antihypertensive regimens on LA functional parameters could not be excluded. The methodology in the strain measurements was based on tissue Doppler imaging, which is immune to angle dependency; however, we took meticulous care to set the region of interest as close to less than 20 degrees against the Doppler beam as possible. As shown in Figure 1, in our experience, the myocardial region around the posterior LA wall appeared to be most suitable for analysis. Finally, the current data of the LA function in HCM patients were derived from one point-measurement. A longitudinal study is necessary to further understand the natural history and reversibility of LA myocardial function in association with medical therapy.

\section{Conclusions}

We assessed LA dyssynchrony during the cardiac cycle using myocardial strain imaging in patients with HCM. About a half of the patients were shown to have LA dyssynchrony in the reservoir phase; that in the emptying phase was less common. HCM patients with LA dyssynchrony were associated with more severe LV hypertrophy, LA enlargement, and LA systolic dysfunction suggestive of significant myocardial involvement.

\section{References}

1. Nagueh SF, Mahmarian JJ. Noninvasive cardiac imaging in patients with hypertrophic cardiomyopathy. J Am Coll Cardiol 2006; 48: 2410-22.

2. Yang H, Woo A, Monakier D, et al. Enlarged left atrial volume in hypertrophic cardiomyopathy: a marker for disease severity. J Am Soc Echocardiogr 2005; 18: 1074- 
82.

3. Sanada H, Shimizu M, Shimizu K, Kita Y, Sugihara N, Takeda R. Left atrial afterload mismatch in hypertrophic cardiomyopathy. Am J Cardiol 1991; 68: 1049-54.

4. Shin MS, Fukuda S, Song JM, et al. Relationship between left atrial and left ventricular function in hypertrophic cardiomyopathy: a real-time 3-dimensional echocardiographic study. J Am Soc Echocardiogr 2006; 19: 796-801.

5. D'Andrea A, Caso P, Romano S, et al. Different effects of cardiac resynchronization therapy on left atrial function in patients with either idiopathic or ischemic dilated cardiomyopathy: a two-dimensional speckle strain study. Eur Heart J 2007; 28: 2738-48.

6. Telagh R, Hui W, Abd El Rahman M, Berger F, Lange $\mathrm{PE}$, Abdul-Khaliq H. Assessment of regional atrial function in patients with hypertrophic cardiomyopathies using tissue Doppler imaging. Pediatr Cardiol 2007 (Epub ahead of print)

7. Di Salvo G, Caso P, Lo Piccolo R, et al. Atrial myocardial deformation properties predict maintenance of sinus rhythm after external cardioversion of recent-onset lone atrial fibrillation. Circulation; 2005; 112: 387-95.

8. Thomas L, McKay T, Byth K, Marwick TH. Abnormalities of left atrial function after cardioversion: an atrial strain rate study. Heart 2007; 93: 89-95.

9. Bax JJ, Abraham T, Barold SS, et al. Cardiac resynchronization therapy: part 1- issues before device implantation. J Am Coll Cardiol 2005; 46: 2153-67.

10. Ypenburg C, Schalij MJ, Bleeker GB, Steendijk P, Boersma E, Dibbets-Schneider P, et al. Impact of viability and scar tissue on response to cardiac resynchronization therapy in ischaemic heart failure patients. Eur Heart J 2007; 28: 33-41.

11. Bleeker GB, Kaandorp TA, Lamb HJ, et al. Effect of posterolateral scar tissue on clinical and echocardiographic improvement after cardiac resynchronization therapy. Circulation 2006; 113: 926-8.

12. Sutherland GR, Di Salvo G, Claus P, D’hooge J, Bijnens B. Strain and strain rate imaging: a new clinical approach to quantifying regional myocardial function. J Am Soc Echocardiogr 2004; 17: 788-802.

13. D'hooge J, Heimdal A, Jamal F, et al. Regional strain and strain rate measurements by cardiac ultrasound: principles, implementation and limitations. Eur J Echocardiogr 2000; 13: 154-70.

14. Breithardt OA, Stellbrink C, Herbots L, et al. Cardiac resynchronization therapy can reverse abnormal myocardial strain distribution in patients with heart failure and left bundle branch block. J Am Coll Cardiol 2003; 42: 48694.
15. Yu CM, Gorcsan J 3rd, Bleeker GB, et al. Usefulness of tissue Doppler velocity and strain dyssynchrony for predicting left ventricular reverse remodeling response after cardiac resynchronization therapy. Am J Cardiol 2007; 100: 1263-70.

16. Devereux RB and Reichek N. Echocardiographic determination of left ventricular mass in man: anatomic validation of the method. Circulation 1977; 55: 613-8.

17. Nagueh SF, Middleton KJ, Kopelen HA, Quinones MA. Doppler tissue imaging: a noninvasive technique for evaluation of left ventricular relaxation and estimation of filling pressures. J Am Coll Cardiol 1997; 30: 1527-33.

18. Abhayaratna WP, Seward JB, Appleton CP, et al. Left Atrial Size: Physiologic Determinants and Clinical Applications J Am Coll Cardiol 2006; 47: 2357-63.

19. Haghjoo M, Bagherzadeh A, Fazelifar AF, et al. Prevalence of mechanical dyssynchrony in heart failure patients with different QRS durations. Pacing Clin Electrophysiol 2007; 30: 616-22.

20. Yu CM, Zhang Q, Yip GW, et al. Diastolic and systolic asynchrony in patients with diastolic heart failure: a common but ignored condition. J Am Coll Cardiol 2007; 49: 97-105.

21. Mollema SA, Liem SS, Suffoletto MS, et al. Left ventricular dyssynchrony acutely after myocardial infarction predicts left ventricular remodeling. J Am Coll Cardiol 2007; 50: 1532-40.

22. Simek CL, Feldman MD, Haber HL, Wu CC, Jayaweera AR, Kaul S. Relationship between left ventricular wall thickness and left atrial size: comparison with other measures of diastolic function. J Am Soc Echocardiogr 1995; 81: 37-7.

23. Maron BJ, Roberts WC. Quantitative analysis of cardiac muscle cell disorganization in the ventricular septum of patients with hypertrophic cardiomyopathy. Circulation 1979; 59: 689-706.

24. Nagakura T, Takeuchi $M$, Yoshitani $H$, et al. Hypertrophic cardiomyopathy is associated with more severe left ventricular dyssynchrony than is hypertensive left ventricular hypertrophy. Echocardiography 2007; 247: 677-84.

25. Oki T, Oishi Y, Tanaka H, et al. Renewed interest in left atrial function: what do we need to evaluate clinically? J Echocardiography 2005; 3: 60-76.

26. Miyoshi H, oishi Y, Mizuguchi Y, et al. Relation of atrial function to ventricular filling during preload reduction in normal subjects: combined analysis of atrioventricular and venous flow velocities. J Echocardiography 2007; 5: 48-54. 